\title{
Diseño de un módulo de bahareque autoconstructivo de bajo costo e impacto ambiental para viviendas unifamiliares
}

Dr. Jorge Luis Rodríguez Ruiz, Mexicano.

ORCID: 0000-0003-4313-5346

jlrr_82@hotmail.com

Instituto Tecnológico Superior del Occidente del Estado de Hidalgo

Cintia Grisel Castañeda Hernández, Mexicana.

ORCID: 0000-0002-2512-4822

cgch1997@ hotmail.com

Instituto Tecnológico Superior del Occidente del Estado de Hidalgo

Rubén Cruz López, Mexicano.

ORCID: 0000-0002-3572-79X

cruzruben910@gmail.com

Instituto Tecnológico Superior del Occidente del Estado de Hidalgo

Mtro. Rogelio Neria Hernández, Mexicano.

ORCID: 0000-0003-0675-0243

neria@itsoeh.edu.mx

Instituto Tecnológico Superior del Occidente del Estado de Hidalgo

Recibido: 01de septiembre del 2020

Aceptado: 06 de febrero del 2021

\section{Resumen}

La necesidad de una vivienda digna, sustentable y autocronstruible es un requerimiento emergente en Mixquiahuala de Juárez, Hidalgo, ya que el 90\% de sus habitantes tienen casas que ellos mismos edificaron. Dichas obras en muy pocos casos están realizadas por algún profesional de la construcción, lo que puede provocar fallas estructurales, espacios inadecuados, problemas de normatividad y de gestión. Esto puede solucionarse por medio de guías o manuales de autoconstrucción que permitan a las personas edificar a sus posibilidades. En este sentido, uno de los elementos que permite la configuración de los espacios son los muros, específicamente los divisorios; estos, por lo regular no se contemplan para ser removidos, dado que son parte importante del sistema estructural de la vivienda. Si se pensará la vivienda con un sistema de planta libre, con muros divisorios removibles, permitiría a los usuarios adaptar la vivienda según las nuevas necesidades. En el mercado existen materiales que permiten construir muros divisorios como la tabla roca, el panel W y el muro a Capuchino (tabique rojo); sin embargo, todos tienen una marcada huella ambiental, 
ya sea por su proceso de elaboración y/o por su traslado. En este sentido, el uso de bajareques se ha convertido en una forma de reducir costos en la construcción de muros divisorios ya que la materia prima de éstos es básicamente la tierra, la madera y el carrizo, convirtiéndolo en un elemento competitivo en términos de costo. Aunado a esto, la huella ambiental para su fabricación es casi nula. Con base en lo anterior, el presente trabajo pretende exponer la elaboración de un módulo de bajareque autoconstruible para una vivienda unifamiliar; de bajo costo, de fácil elaboración y con baja huella ambiental. Este proyecto implicó un proceso de tres años de enseñanza-aprendizaje en conjunto con dos estudiantes que, desde servicio social y posteriormente en su residencia profesional para concluir con la elaboración de su tesis, desarrollaron un prototipo que lograra el reconocimiento sobre temas de la arquitectura hecha con tierra, sobre sistemas constructivos vernáculos y específicamente sobre el bajareque. Estos temas no están en la retícula de la carrera, por lo cual nos vemos en la necesidad de retomarlos en la última etapa de la carrera, mediante del área de investigación, y buscar que con estos proyectos tengan los conocimientos básicos para emprender un posgrado.

\title{
Palabras claves
}

Valor cultural, Autóctono, Medio ambiente, Desarrollo sostenible, Didáctica

\section{Design of a self-constructing bajareque module with low cost and low environmental impact for single-family homes}

\begin{abstract}
The need for a decent, sustainable and self-constructible home is an emerging requirement in Mixquiahuala de Juárez, Hidalgo, since $90 \%$ of its inhabitants have houses that they built themselves. These works in very few cases are carried out by a construction professional, which can cause structural failures, inadequate spaces, regulatory and management problems. This can be solved by means of guides or self-construction manuals that allow people to build to their possibilities. In this sense, one of the elements that allows the configuration of spaces are the walls, specifically the partitions; these are usually not contemplated for removal, since they are an important part of the structural system of the home. If the house is thought of with


an open plan system, with removable dividing walls, it would allow users to adapt the house according to new needs. In the market there are materials that allow the construction of dividing walls such as the rock panel, the W panel and the Capuchino wall (red partition); however, all of them have a marked environmental footprint, either due to their manufacturing process and / or their transfer. In this sense, the use of bajareques has become a way to reduce costs in the construction of dividing walls since the raw material for these is basically earth, wood and reed, making it a competitive element in terms of cost . In addition to this, the environmental footprint for its manufacture is almost nil. Based on the above, the present work aims to expose the development of a self-build low-rise module for a singlefamily home; low cost, easy to manufacture and with a low environmental footprint. This project involved a three-year teaching-learning process in conjunction with two students who, from social service and later in their professional residence to conclude with the preparation of their thesis, developed a prototype that would achieve recognition on issues of architecture made with land, on vernacular construction systems and specifically on the bajareque. These topics are not on the career grid, so we find ourselves in the need to take them up again in the last stage of the career, through the research area, and seek that with these projects they have the basic knowledge to undertake a postgraduate degree.

Keywords: Cultural value, Indigenous, Environment, Sustainable development, Didactics

\section{Introducción}

Con el aumento de la población se da un incremento en la necesidad y construcción de viviendas, un ejemplo es lo que sucede en el Municipio de Mixquiahuala de Juárez, Hidalgo, el cual, según la encuesta del Instituto Nacional de Estadística y Geografía (INEGI) en 2010 pasó de tener 35,200 habitantes en 1995 a 42,834 habitantes en 2010 lo que implica un incremento de la población del $21 \%$ en tan sólo 15 años. Sin embargo, la construcción de viviendas sólo creció 3\%, en el año 2000 existían 7,842 hogares, para 2010 aumentó a 10,514 [1]. Este "incremento" en la construcción de viviendas ha promovido la demanda de materiales para la construcción, como el block y cemento, y esto a su vez trae como consecuencia la explotación de minas para obtener materias primas como la arena y la grava en la región del Valle del Mezquital, Hidalgo, [2] lo cual produce un impacto negativo en el 
medio ambiente, debido a la extracción y transformación de los materiales y el traslado de estos.

Estudios realizados por la Universidad Nacional Autónoma de México (UNAM) revelan que la industria de la construcción es de los mayores sectores generadores de $\mathrm{CO}_{2}$ con $50 \%$, debido al uso de combustibles fósiles y el alta demanda energética en el proceso de construcción [3]. Durante la última década, en diversas localidades clasificadas como marginadas en la región del Valle del Mezquital, existen muy pocos casos de sistemas vernáculos con arquitectura de tierra. Si bien, estas viviendas iniciaron siendo vernáculas, debido al incremento de la demanda, el crecimiento familiar, la falta de mano de obra especializada o el desconocimiento en el tiempo de construcción, necesitaron ampliaciones y sus habitantes recurrieron a la autoconstrucción por medio del sistema tradicional de block y mortero. Esto, por lo regular se da sin una planificación.

En encuestas realizadas en algunas zonas marginadas de Mixquiahuala, muestran que el $100 \%$ de los encuestados no recurrieron a un arquitecto lo que puede provocar disfuncionalidad en la distribución de los espacios, espacios sin ventilación o iluminación natural y/o fallas estructurales en losas y muros lo que implica riesgos para los habitantes, sin embargo, se apoyaron de albañiles, familiares y amigos para edificar su vivienda. En este mismo estudio se registró que todas las viviendas empleaban sistemas constructivos convencionales (block y concreto), sin considerar como una opción la construcción con sistemas vernáculos, un poco por falta de conocimiento sobre la elaboración, los beneficios climáticos y de costo; aunado a esto, hay una clara pérdida de la cultura y tradiciones mexicanas; incluso en La carta del patrimonio vernáculo construido, ratificada por la $12^{\mathrm{a}}$ Asamblea General en México de ICOMOS en octubre de 1999 [4], considera como una línea de acción la conservación e implementación de los sistemas tradicionales de construcción.

Por esta razón; el presente trabajo pretende describir el proceso de elaboración de un sistema constructivo vernáculo a base de tapial y bahareque, que sea estructuralmente estable y constructivamente sustentable (económico y de reducido impacto ambiental) para viviendas de bajos recursos. 
El presente trabajo se basó en una metodología teórico-experimental, donde en principio se investigó sobre las bases teóricas de temas como la arquitecta de tierra, viviendas vernáculas y sistemas constructivos vernáculos; posteriormente se realizaron una serie de pruebas para la realización del módulo de bahareque; y con ello soportar la idea de que este tipo de sistemas son eficaces económicamente y tienen un reducido impacto ambiental en comparación con una vivienda tradicional de block autoconstruida.

\section{Materiales y métodos}

Como ya mencionó, el presente trabajo se basó en una metodología teórico-experimental, por lo cual se iniciará con una breve aproximación teórica, para posteriormente describir el proceso metodológico de la elaboración del bahareque.

\section{Marco conceptual}

La autoconstrucción resurge como solución a la falta de vivienda y carencia de recursos para la adquisición de una por los medios tradicionales (contratación de equipo especializado y/o crédito hipotecario). Según Sergio A. M. (2006) [5]; entre 40\% y 60\% de los parques de vivienda en Latinoamérica son autoconstruidos y tan solo $10 \%$ de esta población recibe atención profesional durante el proceso de edificación de sus viviendas.

La arquitectura vernácula es un sistema constructivo basado en la tradición y la identidad; en este sentido Jocelyn Tillería González lo describe como "un sistema social y cultural complejo, que nace de la relación hombre-entorno, y que refleja de una forma directa, las maneras de habitar" [6]. Se trata de una vivienda que, a pesar o por ser sistemas constructivos de antaño y autóctonos, logran satisfacer las necesidades de nuestro siglo XXI.

Para la construcción de una vivienda a base de tierra, es necesario analizar el tipo de tierra que se tiene, la cual se divide en estratos según la profundidad y sus características; como se representa en el esquema de Gatti, Figura 1. 


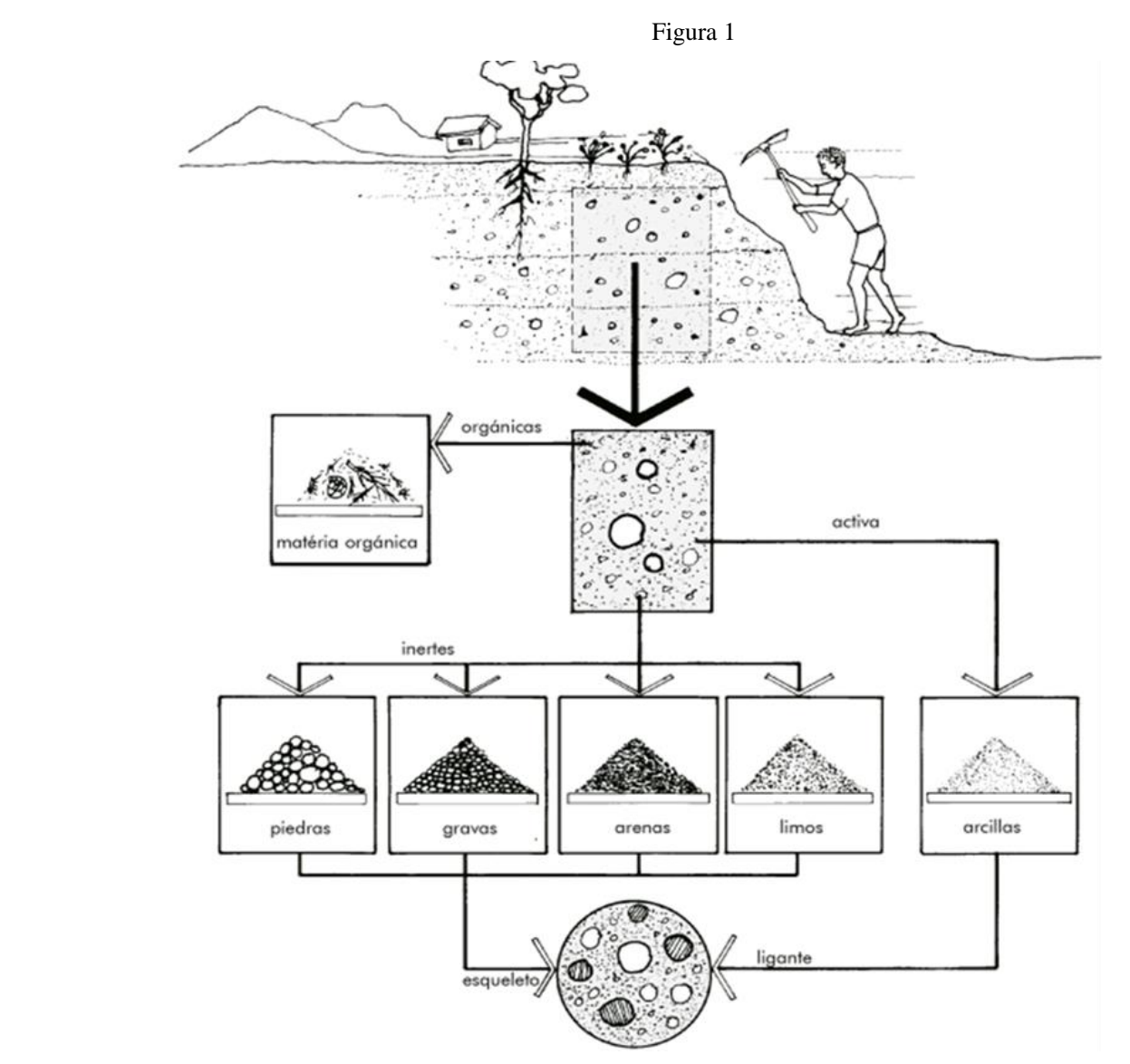

Esquema de Gatti de la División de los estratos y sus características

Fuente: Estudio Comparativo de los Materiales, Productos y Técnicas Contemporáneas en Tierra. Arq. Fabio Gatti.

La norma UNE 103204:1993 [7] para la determinación del contenido de materia orgánica oxidable de un suelo por el método del permanganato potásico, nos indica que se debe rechazar cualquier tierra que contenga cantidades iguales o mayores a $2 \%$ de materia orgánica. Además, se deberán rechazar los suelos que tengan cantidades iguales o mayores a 2\% de sales solubles según la norma UNE 103205:2006 [8] la cual normaliza la determinación del contenido de sales solubles de un suelo.

Los componentes de la "tierra" son: Arcilla que está compuesta por sedimentos detríticos naturales como minerales de grano fino (filosilicatos) principalmente, entre los cuales se encuentra ilita, montmorillonita, clorita, caolinita e interestratificados; y algunos minerales gruesos como: el cuarzo, los feldespatos (alcalinos y plagioclasas), los carbonatos (calcita y dolomita), el yeso y los óxidos de hierro. (Linares G. J., Huertas G. F., Capel M.J. (1983)) [9]. Otro componente es el Limo, sedimento natural; que no posee características 
coloides, al encontrarse en un estado seco carece de cohesión y la resistencia a la fricción es reducidamente menor a las arenas Linares G. J et al., (1983) [9].

La arena está constituida por minerales estables, en estado seco carecen de cohesión; las propiedades de la arena son opuestas a la arcilla, la arena carece de capacidad plástica según Gisbert Blanquer et al., (2010) [10]. Finalmente, la Grava es el componente más estable al estar en contacto con agua, esta carece totalmente de capacidades cohesivas, elásticas, comprensivas y de capilaridad.

\section{Pruebas de la tierra}

Para determinar la calidad de la tierra y el tipo de suelo con el que contamos, se requiere realizar una serie de pruebas de campo para conocer el porcentaje de los componentes. Es importante usar tierra debajo del Horizonte $\mathrm{O}$, para que las pruebas den los resultados apropiados. Toda la tierra que se emplee para la construcción debe ser tamizada previamente figura 2.

Figura 2 Tamizado para obtener la tierra para pruebas (2018). Fuente: Elaboración propia.

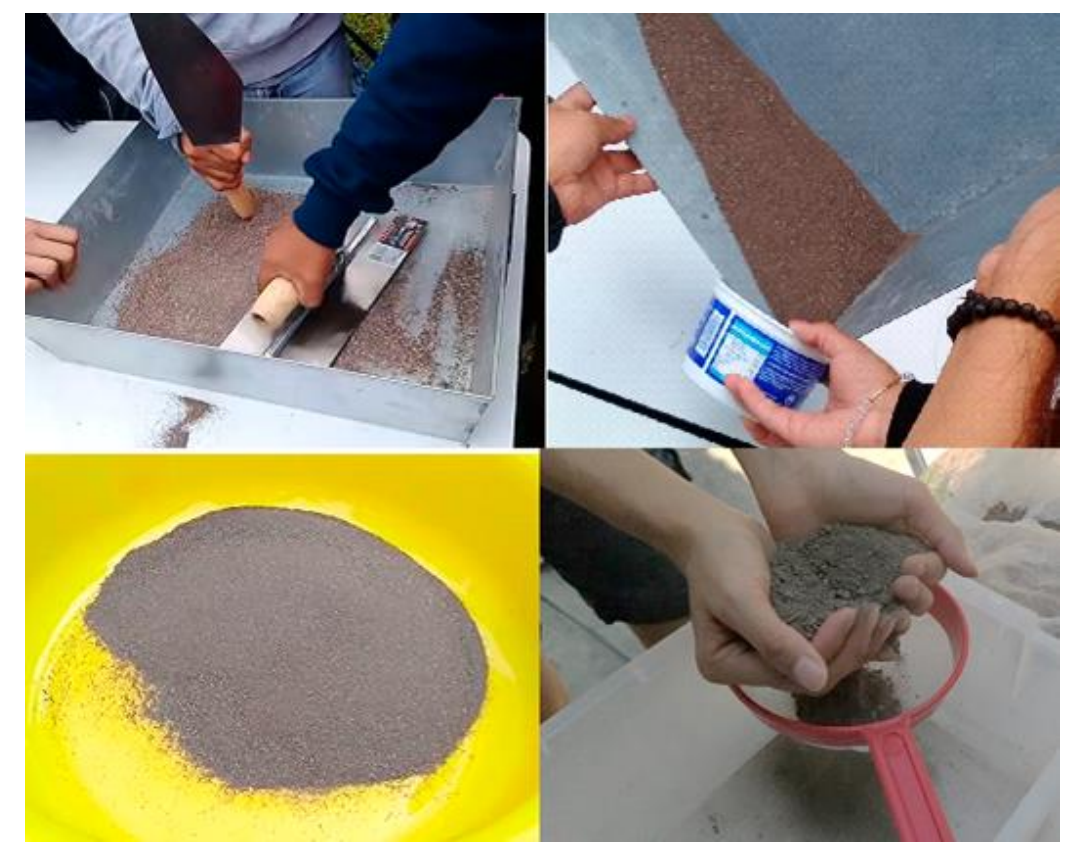




\section{Sedimentación o prueba de botella}

Esta prueba ayuda a detectar las proporciones por medio de la sedimentación, separando en capas los diferentes según su peso propio Figura 3.

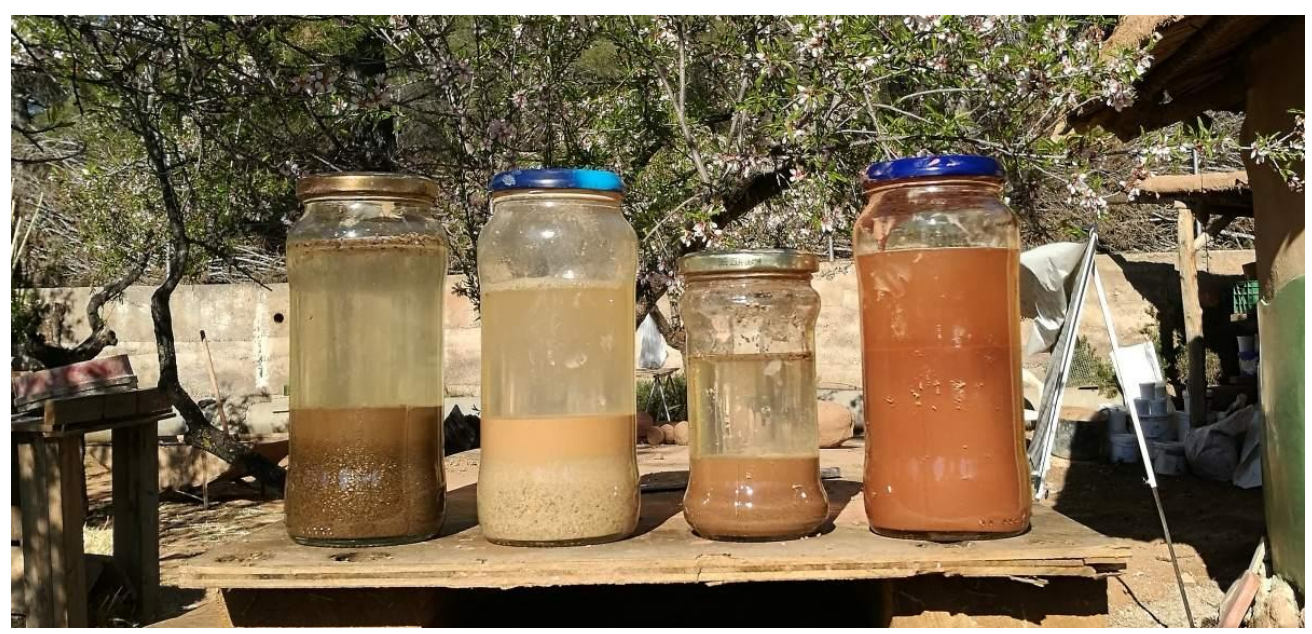

Figura 3 Prueba de botella. Fuente: https://www.arquitecturaysalud.com/bioconstruccion/principios-debioconstruccion (consultada el 9 de febrero de 2020)

\section{Proceso:}

1. A continuación, se describe el proceso de la prueba de sedimentación

1. Se verifica que el frasco esté seco y limpio.

2. Se le coloca masking tape al frasco de la base hasta la boca, esto funcionará para determinar la cantidad de gravas, gravillas, arena, limos y arcillas.

3. Se vierte tierra hasta la mitad de la capacidad de la botella y marcar en el masking tape donde llega la tierra con la letra "T" de Total.

4. Se rellena lo sobrante de la botella con agua dejando espacio suficiente para que pueda agitarse y se tapa.

5. Se agita vigorosamente para que todas las partículas estén suspendidas en el agua, aproximadamente tarda unos 15 minutos.

6. Colocar la botella inmediatamente sobre una mesa o superficie sólida, enseguida se cuentan 15 segundos que es lo que tarda en asentarse las gravas y la arena, en donde se note esta capa, se marca con una línea.

7. Pasados los 15 minutos se podrán ver los limos y se marca con una línea en donde se encuentra la capa. 
8. Se dejará reposar la botella 24 horas para que se sedimente totalmente la arcilla, el agua de la botella debe quedar transparente.

9. Pasado este tiempo se nota claramente hasta dónde llega la arcilla y se coloca una línea. Sí queda flotando algo en el agua, es la materia orgánica.

10. Se mide la distancia entre capas y se anotan en una tabla las proporciones de componentes de la tierra en la columna. Esta distancia entre campas se pone en la columna "Medida (cm).

11. Para sacar el porcentaje que hay de cada elemento se sacará por regla de 3 . Se dividirá la medida en $\mathrm{cm}$ de la capa a sacar entre la medida en $\mathrm{cm}$ de la cantidad de tierra total existente. Si la arcilla se lleva el mayor porcentaje y es más de un $20 \%$, la tierra es muy arcillosa y se requiere integrar arena. Si la tierra es muy arcillosa se requiere agregar arena, si por el contrario es muy arenosa será necesario agregar arcilla a nuestra mezcla para que el material funcione estructuralmente y se eviten grietas o daños estructurales importantes. Con respecto a las proporciones ideales entre los componentes, en la Tabla I: Proporción de los componentes, se describe al elemento constructivo y el porcentaje máximo y mínimo para su aplicación en adobe, bahareque y tapial.

Tabla I Proporción de los componentes para cada elemento constructivo. Fuente: Elaboración propia (2019).

\begin{tabular}{|l|l|l|l|}
\hline Elemento constructivo & Arcilla & Limo & Arena \\
\hline Adobe & $10 \%-20 \%$ & $15 \%-25 \%$ & $55 \%-70 \%$ \\
\hline Bajareque & $20 \%$ & Máximo 30\% & Min 50\% \\
\hline Tapial & $10 \%-30 \%$ & $70 \%-90 \%$ \\
\hline
\end{tabular}

\section{Prueba de plasticidad (Prueba de churro)}

A continuación, se describe el proceso para la realización de la prueba de plasticidad o de churro, esta permite determinar la capacidad plástica que tiene la tierra. 
Proceso:

1. Se colocan $100 \mathrm{~g}$ o un vaso de tierra y se le atomiza agua, para que se humedezca paulatinamente. Usar el atomizador sirve para controlar la humedad del agua debido a que sí se pasa ya no se podrá realizar el churro por la textura de atole que puede llegar a tomar por exceso de humedad. Figura 4.

2. La tierra se humedece hasta lograr una bolita de tierra que no se pegue en las manos y queda como una plastilina.

3. Se amasa la bola sobre una superficie recta hasta lograr un churro de $1.5 \mathrm{~cm}$ de diámetro y más de $30 \mathrm{~cm}$ de largo. Sí el churro se abre mientras se amasa requiere un poco más de agua, pero sí se desmorona significa que es una tierra muy arenosa.

4. Con el metro se mide $25 \mathrm{~cm}$ y se corta el churro

5. Se pasa el churro a una hoja rodándolo para evitar lo más posible su manipulación y se coloca la orilla de la mesa.

6. Se sujeta la hoja y se desliza de forma horizontal, con una mano se sujeta la hoja y con la otra se jala hacia abajo haciendo una forma de cuna con esta mano para sujetar las piezas que se irán cayendo, debido a que sí se cae al piso se requerirá repetir la prueba.

7. Se miden cada pieza que se cortó y sí los cortes tienen una variación de más de 1 $\mathrm{cm}$ significa que la mezcla requiere que se amase más y se repite el procedimiento. Tabla 2. 


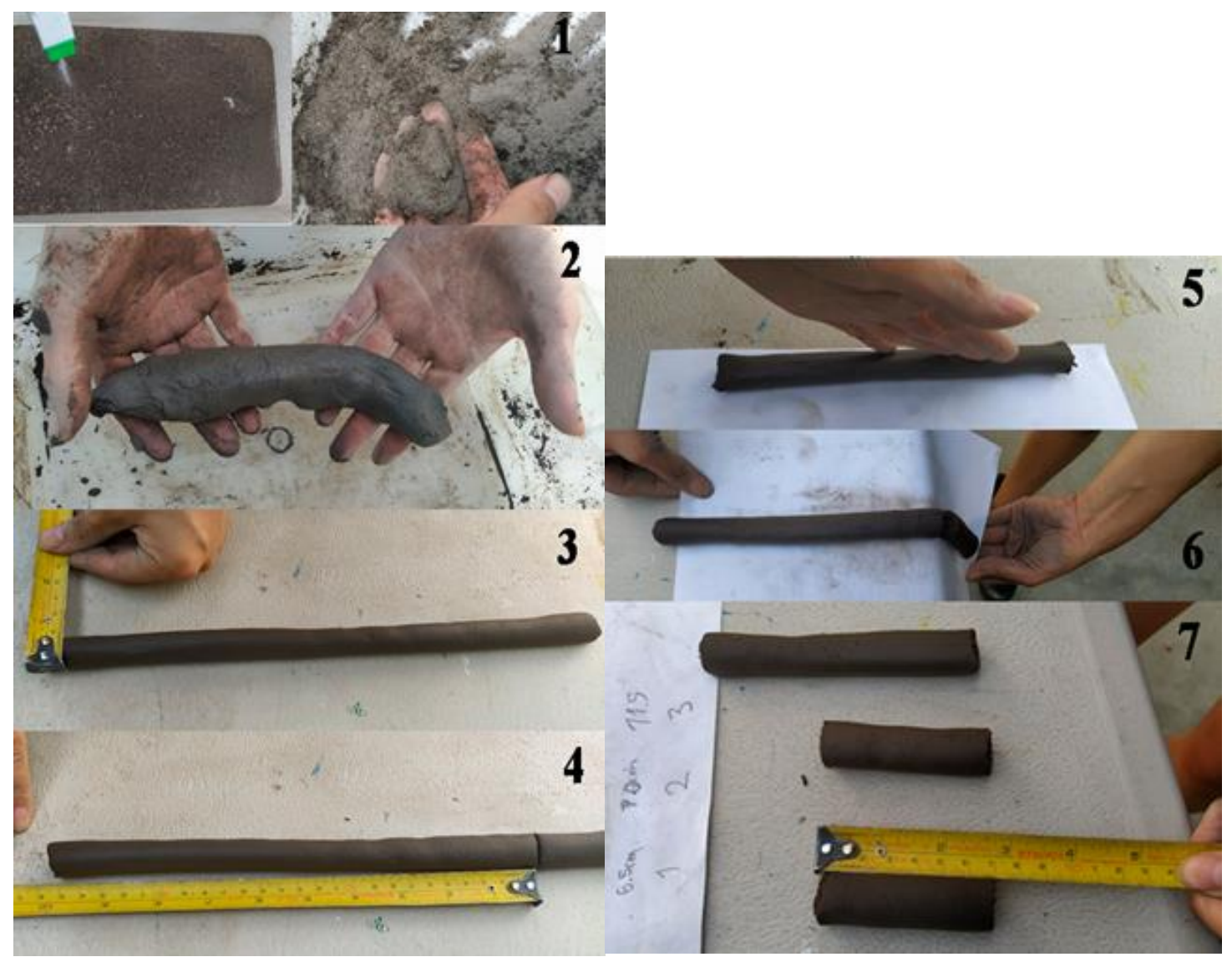

Figura 4. Proceso de prueba de churro. Fuente: Elaboración propia (2018).

Tabla 2. Aplicación según dimensiones de las piezas de churro resultantes. Fuente: Elaboración propia (2019).

\begin{tabular}{|l|l|}
\hline Sistema Constructivo & Medida del churro $(\mathbf{c m})$ \\
\hline Tapial & $0-3$ \\
\hline Adobe & $3-6$ \\
\hline Bajareque y aplanados bases & $6-9$ \\
\hline Pinturas y acabado final & $9-12$ \\
\hline $\begin{array}{l}\text { Mascarillas, alfarería, etc. } \\
\text { No para construcción. }\end{array}$ & +12 \\
\hline
\end{tabular}

Si se requiere hacer un tapial y el churro se cortó a los $5 \mathrm{~cm}$, Figura 5 , se necesita agregar más arena, la tierra del churro se devuelve al molde y se agregan $10 \mathrm{~g}$ de arena. Posteriormente se repite la prueba hasta que se corte a una medida aceptable. 
Sí se requirieron 30 g o $1 / 3$ de vaso para que se corte a $2 \mathrm{~cm}$, que ya entre en el valor para tapial, significa que por cada 3 botes de tierra del sitio se va a necesitar un bote de arena.

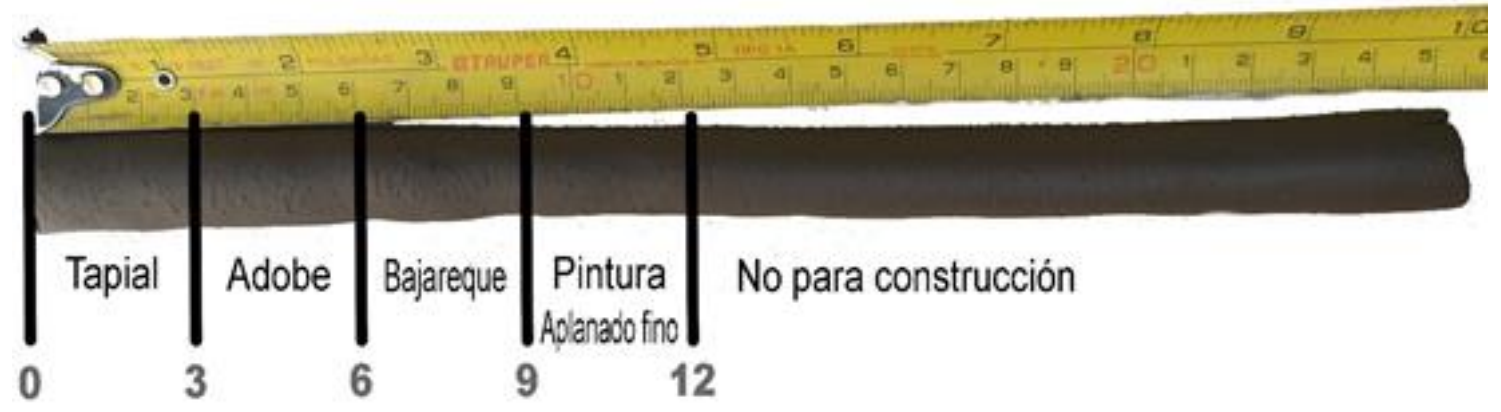

Figura 5. Aplicación de la tierra según el corte del churro. Fuente: Elaboración propia (2018).

\section{Definición del bahareque}

El bajareque o conocido también como quincha, consiste en una estructura en forma de malla de caña, bambú, carrizo o ramas, con una estructura de doble o bastidor, estos son colocados de forma vertical y horizontal y al interior se integra un relleno con una mezcla de barro. Los bajareques tienden a realizarse en paneles rectangulares, el espesor promedio es de $10 \mathrm{~cm}$. Estos paneles se componen por cuatro elementos: estructura maestra, estructura auxiliar, relleno y revestimiento. El bahareque tendrá una medida modulada para todo el proyecto, lo cual facilita el proceso de fabricación y montaje.

\section{Proceso de elaboración del módulo de bahareque}

La aplicación del módulo del bahareque se ejemplifica en una casa chica de $75 \mathrm{~m}^{2}$; aplicando los datos que se describen con relación al costo y a las emisiones de $\mathrm{CO}_{2}$ por kg de materia, este último se retoma de Agüello Méndez [11] aplicándolos al sistema autoconstructivo.

\section{Etapa 1: Armado de muro de bahareque}

Para formar los módulos de bajareque se requiere de un bastidor de igual medida para todas las piezas necesarias. Estos estarán realizados de madera de pino por su calidad, resistencia

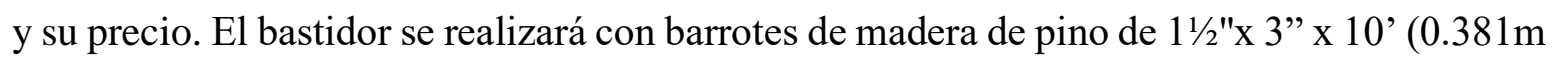
x $7.62 \mathrm{~m}$ x $3.048 \mathrm{~m})$. En el caso de que la madera llegue pandeada se recurrirá a un sistema de 
enderezado. Para la elaboración del bastidor se requiere cortar 2 piezas con una longitud de $0.989 \mathrm{~m}$ y 2 con una longitud de $2.50 \mathrm{~m}$.

Los 2 barrotes de $2.50 \mathrm{~m}$ irán de forma vertical y los 2 barrotes de $0.989 \mathrm{~m}$ se colocarán horizontalmente entre los barrotes verticales, de manera que se oriente el lado más ancho (3") hacia el interior del módulo y el más delgado (11/2”) hacia la cara del bastidor. Por último, se unirán los barrotes por medio de pijas de madera avellanada de $8 \mathrm{~mm}$ x 2 1/2 “. Se colocarán 3 por cada unión, por lo que se emplearán en total 12 pijas de madera. El módulo se rellenará totalmente de tierra, su uso principal es para dividir espacios. Se compone de cuatro molduras (entrepaños internos) con una longitud de $0.989 \mathrm{~m}$

La colocación de divisiones y refuerzos en el módulo 1 se realizará tomando las 2 piezas de moldura unidas a los barrotes del bastidor del lado interno, empleando una pija al centro y una en cada extremo de la moldura, dando en total 3 pijas por moldura, Figura 6. Las otras 2 molduras se distribuirán a lo largo del bastidor, quedando a cada $0.808 \mathrm{~m}$, estos elementos se unirán usando una sola pija por extremo, siendo en total 2, atornillándose desde los lados exteriores, ver figura 6.

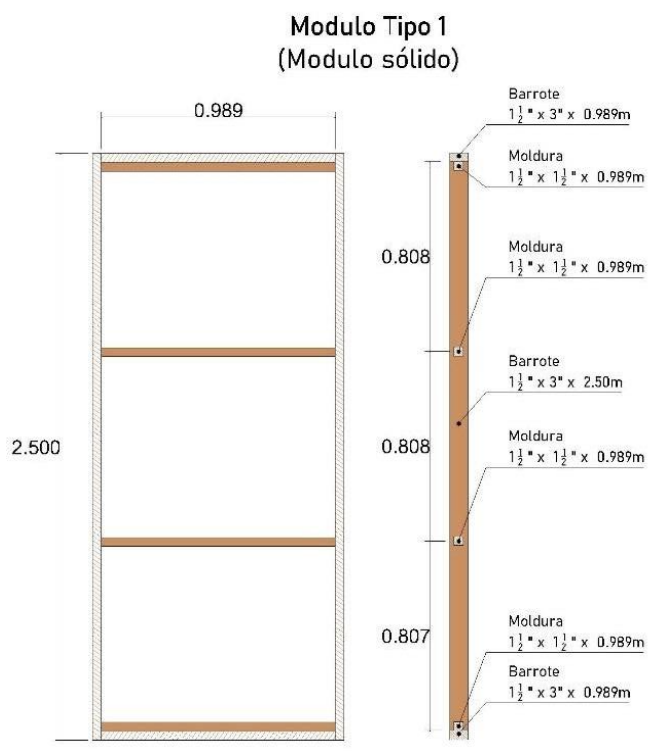

Figura 6. Estructura del módulo. Fuente: Elaboración propia (2019).

\section{Etapa 2. Entramado de los bastidores}


A los bastidores se le agregará un entramado de carrizo. Las piezas de carrizo que se empleen deben tener un diámetro entre $1.5 \mathrm{~cm}$ y $3 \mathrm{~cm}$ de grosor, y de largo $2.50 \mathrm{~m}$ al menos. Para colocar los carrizos en el bastidor se van entrelazando en el bastidor entre las molduras cuadradas, pasándolos de lado opuesto cada vez que llegue a una moldura diferente, produciendo el efecto de un trenzado. Las molduras que están en las bases de los bastidores o al final de la zona de entramado sirven como área para retener los carrizos y así lograr que se tense correctamente, dejando los huecos en cada entrelazado. Cuando los carrizos se vayan ingresando en el bastidor, tendrán un largo mayor a la necesitada en el bastidor, para ello, se recortará el sobrante, Figura 7.

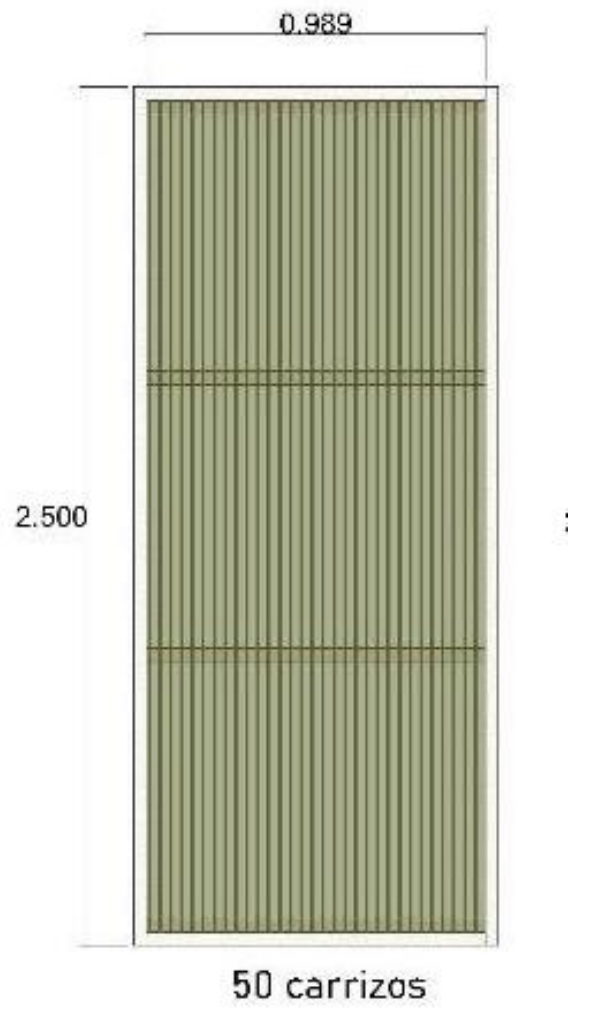

Figura 7. Distribución carrizos en el Módulo tipo. Fuente: Elaboración propia (2019).

Posteriormente se pintarán las piezas de madera con aceite quemado o aceite virgen, como un tratamiento a la madera para evitar daños por humedad. Realizado esto se dejará secar el bastidor con el carrizo al menos 2 días, Figura 8, en un área ventilada sin contacto directo del sol. 


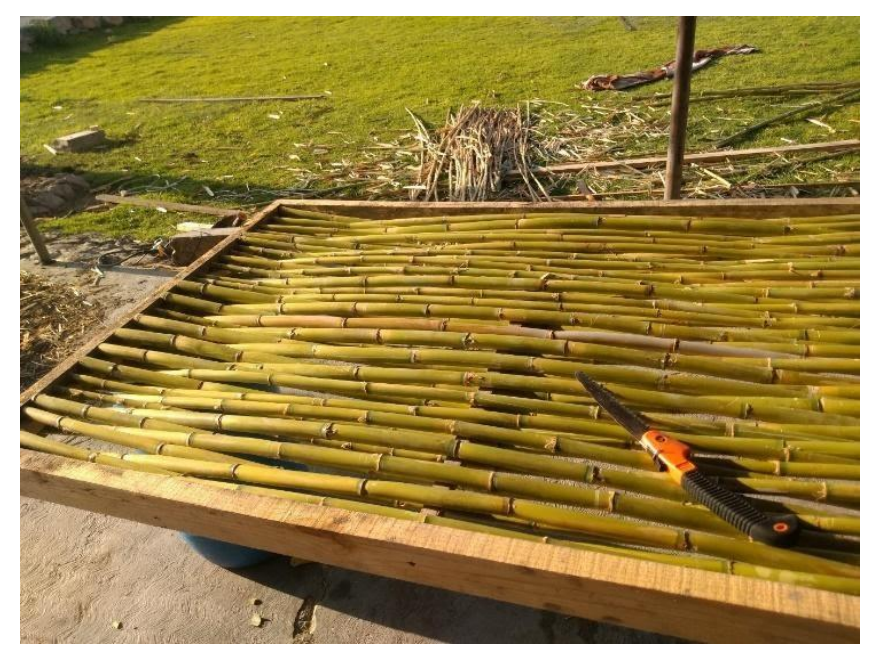

Figura 8. Vista del entramado de carrizo. Fuente: Elaboración propia (2018).

Finalmente, el módulo de bahareque se colocará entre los espacios creados entre los polines, atornillando con pijas del \#8 de 2 1⁄2”, las cuales se colocarán a cada $40 \mathrm{~cm}$ de ambos lados del bastidor.

\section{Etapa 3. Preparación de la mezcla de tierra para rellenar los muros de bajareque}

Para la realización del bahareque, la paja tiene que estar totalmente seca y ser cortada de forma delgada. La tierra en conjunto con la paja debe hidratarse de forma constante y paulatina con una manguera evitando hacer un "volcán" con la mezcla, este proceso se realiza hasta que la tierra haya tomado una consistencia chiclosa y homogénea con la paja, para revolver la mezcla se recomienda el uso de una pala.

La colocación del relleno se realizará iniciando desde la parte baja del bajareque y de allí hasta la parte superior de forma ordenada y pareja. Se realizarán bolas de tierra del tamaño de un puño y se lanzarán al módulo con la suficiente fuerza para que la tierra pase entre los huecos. De vez en cuando se debe modelar con las manos la tierra para verificar que la tierra entre en los huecos o para distribuir los montones de barro que se hagan.

Posteriormente se dejará secar el muro por al menos 48 horas para poder colocar el primer aplanado, la mezcla para aplanado es tierra-cabello en proporción 10:1, por cada 10 botes de tierra se usará 1 de cabello, la textura de la mezcla es chiclosa. Este primer aplanado tiene la función de nivelar el muro y alisarlo para cubrir las imperfecciones originadas por el 
relleno base y tendrá un grosor de aproximadamente $3 \mathrm{~cm}$, Figura 9. Antes de colocar el aplanado, se debe humedecer la superficie del muro de tierra para que ambos materiales tengan mayor adherencia. Cuando se termine de aplicar el aplanado se dejará secar al menos dos horas para poder aplicar otra capa de aplanado, la cual deberá tener un grosor máximo de $1 \mathrm{~cm}$ y se dejará secar otras dos horas.

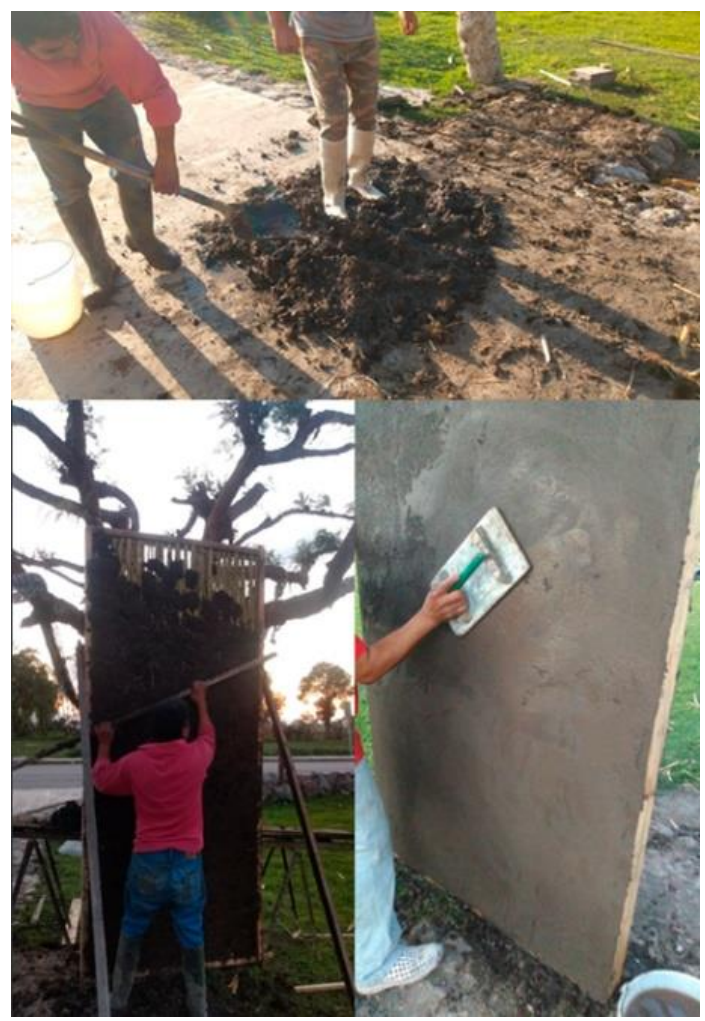

Figura 9 Proceso de preparación y colocación del mortero para los muros de bajareque. Fuente: Elaboración propia (2018).

En la tabla III se demuestra el costo del material para realizar un $\mathrm{m}^{2}$ de bahareque y la emisión de $\mathrm{CO}_{2}$ que tendría por la realización de la vivienda de $75 \mathrm{~m}^{2}$ 
Tabla 3. Costo del bahareque por m2. Fuente: Elaboración propia (2019).

\begin{tabular}{|c|c|c|c|c|c|}
\hline Material & $\mathrm{U}$ & Cant & P.U. & Importe & Emisión de $\mathrm{CO} 2$ por kg de \\
\hline Barrotes & pza & 2 & $\$ \quad 38.00$ & $\$ 76.00$ & .54 \\
\hline Molduras & pza & 2 & $\$ \quad 35.00$ & $\$ 70.00$ & .18 \\
\hline Pijas & pza & 22 & 0.35 & 7.70 & 0.0028 \\
\hline Carrizos & pza & 50 & $\$ \quad 1.00$ & $\$ \quad 50.00$ & 0.00 \\
\hline Gualdras & pza & 1 & $\$ 450.00$ & $\$ 450.00$ & 15 \\
\hline Tierra & $\mathrm{m} 3$ & 0.3 & $\$ 228.60$ & $\$ \quad 68.58$ & .021 \\
\hline Paja & $\mathrm{kg}$ & 3 & \$ $\quad 11.11$ & $\$ 33.33$ & 0.00 \\
\hline Arena & $\mathrm{m}^{3}$ & 0.015 & $\$ 246.42$ & 3.70 & 0.416 \\
\hline \multirow[t]{2}{*}{ Agua } & $\mathrm{L}$ & 80 & 0.28 & $\$ \quad 22.08$ & 0.00 \\
\hline & & & Total & $\$ 781.39$ & \\
\hline
\end{tabular}




\section{Resultados}

A continuación, se describen el costo total y el impacto al medio ambiente de una vivienda con un sistema de autoconstrucción a base de cemento, acero y block en comparación de una vivienda con el uso del módulo del bahareque.

En la tabla 4 se muestra la emisión de $\mathrm{CO}_{2}$ que tendría la realización de la vivienda de $75 \mathrm{~m}^{2}$ a base de block, acero y cemento.

Tabla4. Emisión de $\mathrm{CO} 2$ por Kg de material con el Sistema Autoconstructivo a base de Cemento, Acero y Block. Fuente: Elaboración propia (2019).

\begin{tabular}{|l|l|l|} 
& Cantidad & $\begin{array}{c}\text { Emisión de CO2 por } \\
\text { kg de material }\end{array}$ \\
\hline Cemento Tonelada & 7.25 & 502.14 \\
\hline Tabique piezas & $14000(98000 \mathrm{~kg})$ & 40180 \\
\hline Grava Tons & 10 & \\
\hline Arena Tons & Total de emisiones & $42245.05 \mathrm{Kg} \mathrm{de} \mathrm{CO2}$ \\
\hline Acero Kg & 14 & 0.07 \\
\hline & & 1562 \\
\hline & & \\
\hline & & \\
\hline & & \\
\hline & & \\
\hline
\end{tabular}


En la tabla 5 se demuestra la emisión de $\mathrm{CO}_{2}$ que tendría por la realización de la vivienda de $75 \mathrm{~m}^{2}$ a base de bahareque.

Tabla 5. Emisión de $\mathrm{CO} 2$ por Kg de material con el Sistema Autoconstructivo a base de Bahareque. Fuente: Elaboración propia (2019).

\begin{tabular}{|c|c|c|}
\hline & Cantidad & $\begin{array}{l}\text { Emisión de } \mathrm{CO} 2 \text { por } \mathrm{kg} \\
\text { de material }\end{array}$ \\
\hline Tierra & $3890 \mathrm{~kg}$ & 27.23 \\
\hline Madera & $700 \mathrm{~kg}$ & 42 \\
\hline Acero/Metal & $200 \mathrm{~kg}$ & 560 \\
\hline Arena & $100 \mathrm{~kg}$ & 0.7 \\
\hline Paja & $400 \mathrm{~kg}$ & 0.00 \\
\hline Carrizos & $1380 \mathrm{~kg}$ & 0.00 \\
\hline & Total de emisiones & $629.93 \mathrm{Kg} \mathrm{de} \mathrm{CO}^{2}$ \\
\hline
\end{tabular}


En la tabla 6 se muestra la comparación del costo, de emisiones de $\mathrm{CO}_{2}$ y tiempo de construcción entre una vivienda a base de block, cemento y acero y una vivienda a base de bahareque.

Tabla 6. Comparativa de precios/emisiones de $\mathrm{CO} 2$ y tiempo de construcción. Fuente: Elaboración propia (2019)

\begin{tabular}{|l|l|l|l|l|}
\hline & Costo $\mathrm{m}^{2}$ & Costo $75.9 \mathrm{~m}^{2}$ & $\begin{array}{l}\text { Emisión de } \mathrm{CO}_{2} \\
\text { por Kg de material }\end{array}$ & $\begin{array}{l}\text { Tiempo de } \\
\text { construcción }\end{array}$ \\
\hline $\begin{array}{l}\text { Casa } \\
\text { Tradicional }\end{array}$ & $\$ 8,553.73$ & $\$ 649,228.11$ & $\begin{array}{l}42245.05 \mathrm{Kg} \text { de } \\
\mathrm{CO}^{2}\end{array}$ & 20 sem. \\
\hline $\begin{array}{l}\text { Casa Vernácula } \\
\text { Diferencia de } \\
\text { precio/emision } \\
\text { es/tiempo }\end{array}$ & $\$ 5,394.76$ & $\$ 409,462.14$ & $629.93 \mathrm{Kg} \mathrm{de} \mathrm{CO}{ }^{2}$ & 16 sem. \\
\hline
\end{tabular}

Como se observa, el costo de una vivienda con el sistema autoconstructivo a base de bajareque es $33 \%$ más económico y rápido de construir. Esto se debe principalmente a que con el diseño se buscó facilitar que el bahareque fuera sencillo de realizar. La modulación del bahareque permite la facilidad de la fabricación e instalación de los bahareques.

También se demuestra que la huella ecológica es $90 \%$ menor en el sistema autoconstructivo de bahareque, ya que por el uso de acero y cemento implican un mayor coste energético ya sea por su traslado y/o transformación. Se evidenció a lo largo del texto; que construir con sistemas o elementos vernáculos; como el que se expuso, también promueve la cohesión familiar, ya que el método y técnica requieren de trabajo en equipo, promoviendo lazos de comunión y un mayor apego a la vivienda. 
En la Figura 10 se muestra el resultado del bahareque una vez terminado, en las figuras 11, 12 y 13 se muestran fotografías de cómo se vería la vivienda con la incorporación del bahareque.

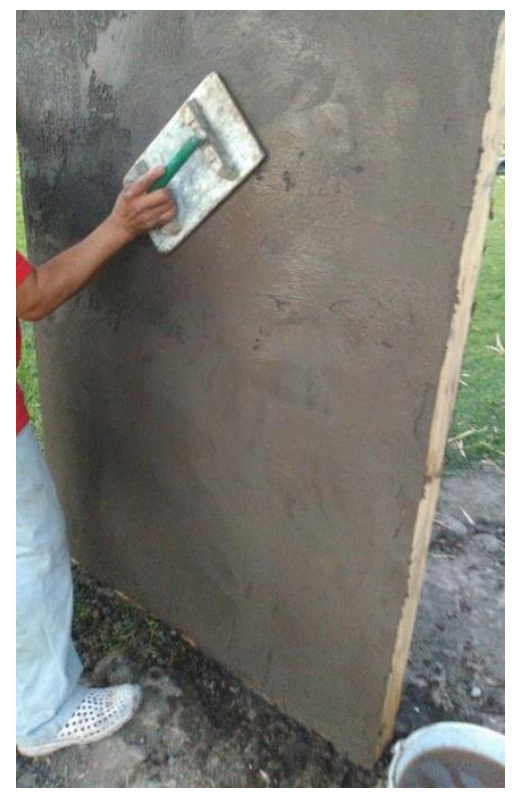

Figura 10. Vista del módulo del bahareque ya terminado. Fuente: Elaboración propia (2018).

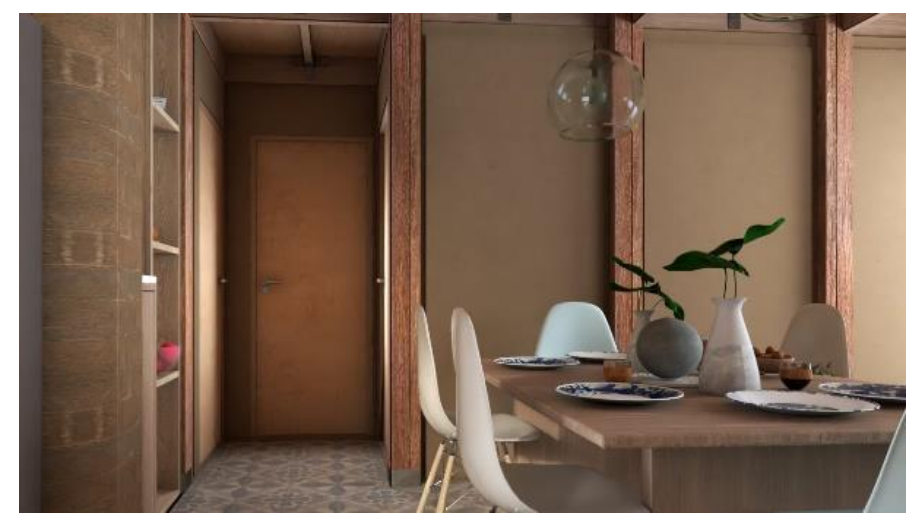

Figura 11. Imagen fotorrealista donde se aprecia la incorporación del bahareque en la sala comedor de la vivienda (2020). 


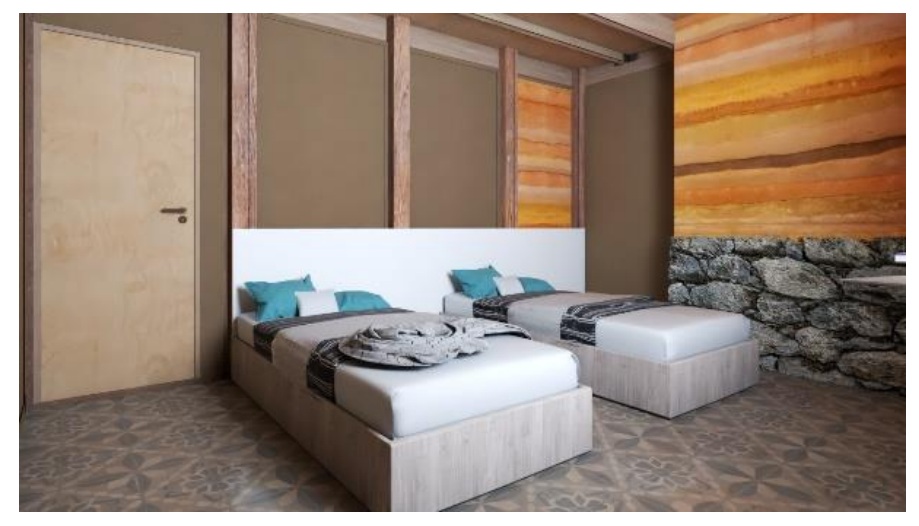

Figura 12. Imagen fotorrealista donde se aprecia la incorporación del bahareque en la recámara secundaria (2020).

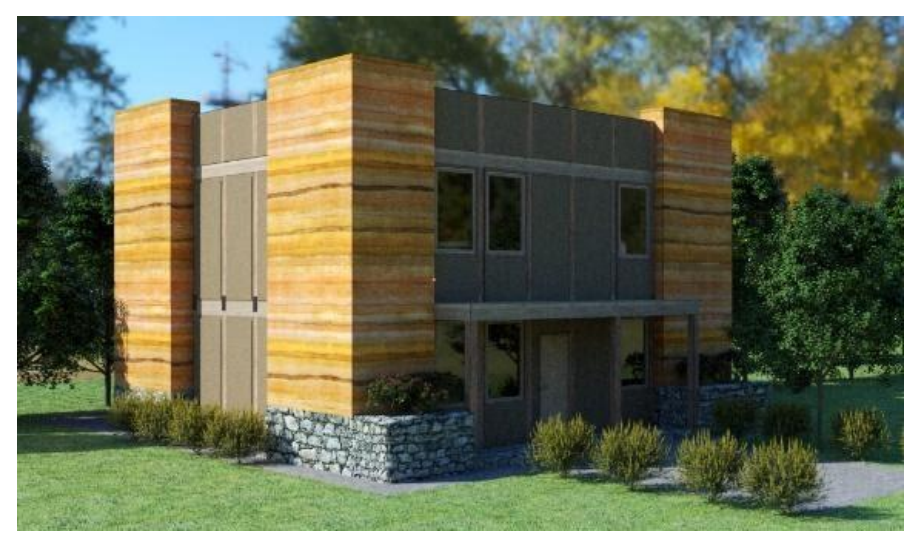

Figura 13. Imagen fotorrealista donde se aprecia la incorporación del bahareque al exterior de la vivienda (2020).

\section{Conclusiones}

Desafortunadamente aún existe cierto recelo hacia los sistemas constructivos y elementos vernáculos, ya que se creen insalubres, costosos y poco prácticos. Aunque hay otras formas de hacer viviendas, es conveniente considerar la combinación de sistemas vinculados con los convencionales y pueden dar muy buenos resultados debido a que se abaratan costos y recursos, además se disminuye el impacto ambiental e incrementa la cultura y la cohesión social de las comunidades. Es así como, los beneficios de la fabricación y el uso de bajareque son: la flexibilidad del uso de un módulo estandarizado, se puede retirar y colar con mucha facilidad y a largo plazo, se desmantela y los restos se pueden utilizar como composta. 
Los profesionales de la construcción deberíamos ser más consientes con nuestras propuestas y comenzar a alejarnos de los vicios de un sistema capitalista, de los monopolios de la industria de la construcción; dejar de ver a la arquitectura sólo como un negocio, y retomar el ideal de la arquitectura que es comprender a las personas y/o futuros clientes, ya que al final para ellos hacemos arquitectura, en particular para aquellas personas de escasos recursos y/o población más vulnerables, que son la mayor parte de la población en México. Es incongruente pensar y hacer arquitectura globalizada en un país con tantas diferencias sociales, es momento de concientizarnos y comenzar a reflexionar cuál debería ser el quehacer del arquitecto con una visión sustentable.

\section{Referencias}

[1] INEGI, Instituto Nacional de Estadística y Geografía (2010) Anuario de Estadística por Entidad Federativa. INEGI.

[2] Neria Hernández, R., Pérez Herrera, L., \& Rodríguez Ruiz, J. (septiembre de 2017). Análisis de la vulnerabilidad del entorno del Municipio de Mixquiahuala de Juárez, Hgo. Revista de Arquitectura y Diseño, 1, 20-30.

[3] (2018). La construcción genera más del 50\% de los contaminantes en el mundo. Digital Bricks recuperado de: https://digitalbricks.com.mx/2018/08/01/la-construccion-genera-masdel-50-de-los-contaminantes-en-el-mundo/s

[4] Consejo Internacional de Monumentos y Sitios. (1999). Carta de Patrimonio Vernáculo Construido. Madrid. Ratificada en México: ICOMOS.

[5] Malatesta, S. A. (2006). Análisis del proceso de autoconstrucción de la vivienda en chile, bases para la ayuda informática para los procesos comunicativos de soporte. Universidad de Catalunya

[6] González, J. T. (2017). La arquitectura sin arquitectos, algunas reflexiones sobre arquitectura vernácula. AUS (Arquitectura/Urbanismo/Sustentabilidad). doi:10.4206/aus.2010.n8-04

[7] UNE-Norma Española. Ediyada e impresa por AENOR, Deposito legal:M7149:2006 
[8] UNE-Norma Española. Ediyada e impresa por AENOR, Deposito legal:M7149:2006

[9] Linares González, J., Huertas García, F., \& Capel Martínez, J. (1983). La arcilla como material cerámico. Características y comportamiento. Cuadernos De Prehistoria Y Arqueología De La Universidad De Granada. Disponible en: https://revistaseug.ugr.es/index.php/cpag/article/view/122

[10] Gisbert Blanquer ,J.M, Ibañez Asensio S.,\& Moreno Ramón ,H. (2010). La textura del suelo.

[11] Argüello Méndez, T. d., \& Cuchí Burgos, A. (enero-marzo de 2008). Análisis del impacto ambiental asociado a los materiales de construcción empleados en las viviendas de bajo coste del programa 10 x10 Con Techo-Chiapas del CYTED. Informes de la Construcción, 60(509), 25-34. 